\title{
Volume versus standard coils in the treatment of intracranial aneurysms
}

\author{
Johannes Kaesmacher, ${ }^{1}$ Christina Müller-Leisse, ${ }^{1}$ Thomas Huber, ${ }^{1}$ \\ Tobias Boeckh-Behrens, ${ }_{1}^{1}$ Bernhard Haller, ${ }^{2}$ Ehab Shiban, ${ }^{3}$ Benjamin Friedrich, ${ }^{4}$ \\ Claus Zimmer, ${ }^{1}$ Franziska Dorn, ${ }^{5}$ Sascha Prothmann ${ }^{1}$
}

\begin{abstract}
${ }^{1}$ Abteilung für diagnostische und interventionelle Neuroradiologie, Klinikum rechts der Isar, Technische Universität München, Munich, Germany

${ }^{2}$ Institut für Statistik und Epidemiologie (IMSE), Klinikum rechts der Isar, Technische Universität München, Munich, Germany

${ }^{3}$ Klinik für Neurochirurgie, Klinikum rechts der Isar, Technische Universität München, Munich, Germany ${ }^{4}$ Klinik und Poliklinik für Diagnostische und Interventionelle Radiologie, Universitätsklinikum Leipzig, Leipzig, Germany

${ }^{5}$ Abteilung für Neuroradiologie, Klinikum Großhadern, LudwigMaximilians-Universität München, Munich, Germany
\end{abstract}

\section{Correspondence to} Johannes Kaesmacher, Abteilung für diagnostische und interventionelle Neuroradiologie, Technische Universität München, Ismaninger Str 22, Munich 81675, Germany;

j.kaesmacher@tum.de

FD and SP contributed equally.

Received 14 August 2015 Accepted 28 September 2015 Published Online First 21 October 2015

\begin{abstract}
Background Volume coils were developed to improve occlusion rates of intracranial aneurysms. Previous studies have shown increased packing density and comparable occlusion rates, but subgroup analyses of aneurysm size have not been carried out.

Objective To evaluate the safety and efficacy of the Penumbra Coil 400 (PC400) system in treating intracranial aneurysms compared with standard diameter coils.

Methods A monocentric retrospective case review of 260 aneurysms in 233 patients was carried out. In 37 aneurysms the PC400 system was used, while 223 aneurysms were treated with conventional coils. Previously treated aneurysms and aneurysms treated with flow diverters were excluded. Aneurysm and procedure characteristics, packing density, postprocedural and follow-up occlusion grades as well as coil compaction were evaluated.
\end{abstract}

Results Aneurysms treated with PC400 coils had higher volume (218.9 vs $\left.47.1 \mathrm{~mm}^{3}, p<0.001\right)$, wider necks $(3.0$ vs $2.5 \mathrm{~mm}, p=0.005)$, and greater dome/neck ratio ( 2.0 vs $1.6, p=0.001$ ) in comparison with aneurysms treated with conventional coils. Compared with controls, in the PC400 group we achieved higher packing densities ( $43.2 \%$ vs $34.4 \%, p<0.001$; in aneurysms $\geq 7 \mathrm{~mm}$ $42.2 \%$ vs $27.8 \%, p<0.001)$. On follow-up angiography we observed less coil compaction ( $23.8 \%$ vs $64.3 \%$, $\mathrm{p}=0.003$ ) and less aneurysm recurrence ( $14.3 \%$ vs $40.5 \%, p=0.046)$ in aneurysms $\geq 7 \mathrm{~mm}$ when using the PC400 system.

Conclusions Use of the PC400 system as opposed to conventional coils suggests that the PC400 system is safe and effective in treating intracranial aneurysms. Despite having been applied in a potentially more difficult-to-treat group, the use of PC400 was associated with less coil compaction and aneurysm recurrence in aneurysms $\geq 7 \mathrm{~mm}$.

\section{INTRODUCTION}

Over the past decades endovascular coil embolization has developed into a safe and effective technique in treating intracranial aneurysms. ${ }^{1-5} \mathrm{~A}$ major problem of coil embolization is the recanalization of the aneurysms' lumen over time, which occurs in around 20$30 \% .^{6-9}$ Coil compaction is regarded as one of the major factors which have been implicated in aneurysm recanalization and its occurrence is influenced by the initial packing density. ${ }^{10}{ }^{11}$ Aneurysm recurrence is a risk factor for rebleeding. ${ }^{12}$ In large aneurysms, in particular, it remains difficult to achieve adequate packing densities. $^{713}$ Early studies in the mid-2000s suggested that thicker or more complex shaped coils can achieve higher packing densities. ${ }^{14-16}$ The Penumbra Coil 400 (PC400, Penumbra, Inc, Alameda, California, USA) was introduced in early 2011, and promoted as a 'volume coil'. It is considered to be an alternative coil device, which is especially useful when treating large aneurysms.

The PC400 coil has a 'coil within coil' design, resulting in an intrinsic softness and providing greater volume per unit length owing to its 0.020 inch diameter. ${ }^{17}$ The complex structure consists of a stretch-resistant nitinol wire, an inner structural coil, and an outer thin filament. It is thought to be more resistant to mechanical stress and may enhance coil stability, thus preventing coil compaction. To place PC400 coils a special, wider-diameter delivery microcatheter (PX Slim; distal/proximal outer diameter: $0.867 \mathrm{~mm}=2.6 \mathrm{~F} /$ $0.983 \mathrm{~mm}=2.95 \mathrm{~F}$ ) is required. Initial reported experiences with the PC400 coil were positive and showed higher packing densities, better costeffectiveness, reduced procedure time, and comparable short-term occlusion rates. ${ }^{18-21}$ We hypothesize that large cerebral aneurysms, which are particularly difficult to treat, can be safely occluded using PC400 coils, since better packing can be expected to prevent recurrence of aneurysms and coil compaction.

\section{PATIENTS AND METHODS \\ Patient selection}

We present a monocentric retrospective case series of 260 endovascularly treated ruptured and unruptured cerebral aneurysms in 233 patients. We included all subjects with saccular aneurysms treated by coil embolization between January 2010 and May 2015. Criteria for exclusion were the use of flow diverters, incomplete information about the coils used, inadequately calibrated angiography system for volume calculation, and previously treated aneurysms. The study group consisted of 37 aneurysms treated with unique large-diameter coils, whereas the control group included 223 aneurysms treated with different widely used standard coils. Approval from our institutional review board was obtained for this study.

\section{Endovascular treatment}

In the study group we used PC400 coils alone, or mixed with standard diameter coils, whereas a 
variety of standard diameter coils were used in the control group (Orbit Galaxy, Trufill: Codman \& Shurtleff, Inc, Ranyham, Massachusetts, USA; HyperSoft 3D, HydroCoil, Cosmos, Complex, Compass: Microvention, Inc., Tustin, California, USA; Axium: Covidien, Irvine, California, USA; Target, GDC: Stryker, Fremont, California, USA; diameter range 0.0095-0.015 inch). When mixing coil materials in the study group the conventional coils were used only for neck finishing. The choice of which coil type to use was mainly influenced by aneurysm characteristics, such as volume, neck width, and maximum diameter. An indication for endovascular intervention versus surgical clipping was established based on consensus in our institutional vascular board consisting of experienced interventional neuroradiologists and neurosurgeons. Endovascular coil embolization was performed under general anesthesia and with systemic administration of heparin. The aim of coil placement was defined as packing aneurysms as densely as possible, with coils being incorporated into the aneurysm sac until no more material could be inserted.

\section{Analysis and outcome definitions}

Aneurysmal characteristics were analyzed by reviewing angiographic data. The review was blinded and performed by two experienced neurointerventionalists (TB-B, SP), who were not involved in the treatment of the reviewed case. Aneurysm volume and packing density were calculated using the recently evaluated software, 'AngioSuite'. ${ }^{22}$ Packing density was also qualitatively analyzed using packing density ranges $(0-10 \%, 10-$ $20 \%, 20-30 \%, 30-40 \%$, and $>40 \%$ ). Aneurysms were further divided into diameter categories using the cut-off values proposed by the authors of the ISUIA (International Study of Unruptured Intracranial Aneurysms) trial: small $<7 \mathrm{~mm}$, medium 7-12 mm, large $>12-25 \mathrm{~mm}^{23}$ Dome/neck ratio was defined as the dome diameter divided by the aneurysm neck. To determine procedure success we evaluated grade of occlusion using the modified Raymond-Roy occlusion classification (MRRC) after the initial procedure and based on follow-up images. ${ }^{24}$ Follow-up angiograms were further dichotomized into present or absent compaction, where coil compaction was defined as shrinkage of the coil mesh or increased contrast agent filling within the coil interstices compared with the postinterventional angiogram. Aneurysm recurrence was defined as deterioration of the MRRC to grade IIIa or IIIb on follow-up images. Overall treatment failure was defined as MRRC grade IIIa or IIIb on follow-up images as well as a MRRC grade IIIb after the procedure when no follow-up data were available. Although we are aware that this definition may be controversial, we adopted it based on evidence showing that an aneurysm with an initial occlusion grade of IIIb has very low chances of having a better occlusion rate grade at follow-up control. ${ }^{25} \mathrm{We}$ did not include MRRC grade II as treatment failure because a recent study suggested that grade II aneurysms are comparable to grade I aneurysms in their recanalization and re-treatment rates. ${ }^{18}$ Procedure time was defined as the time between first image acquisition and the first control image. We also classified aneurysms as 'high risk' if they shared at least one of the previously reported shape characteristics associated with an increased risk of aneurysm recurrence: neck width $>4 \mathrm{~mm}$, maximum diameter $>10 \mathrm{~mm}$, mean volume $>600 \mathrm{~mm}^{3} .89132627$

\section{Statistical analysis}

Analysis was performed on all aneurysms, and also after excluding aneurysms with a diameter $<7 \mathrm{~mm}$ to increase comparability of both groups. Continuous variables with a normal distribution were compared using Welch's t test for independent samples, whereas the Mann-Whitney U test was used for nonnormally distributed data. Categorical variables and frequency counts were compared using Fisher's exact test. All noted $\mathrm{p}$ values are two sided. If not otherwise specified, normally distributed variables are shown as mean $\pm \mathrm{SD}$, and other data are reported as median and IQR. To determine the best cut-off values we performed receiver operating characteristic analysis and determined the highest Youden index (sensitivity+specificity-1). Multiple aneurysms seen in the same patient were treated as independent observations. All data management and statistical analyses were performed using SPSS statistics (V.23.0; IBM Co/rporation, Armonk, New York, USA).

\section{RESULTS}

\section{Patient and aneurysm characteristics}

Most patient and aneurysm characteristics of our patients are comparable to those of published large aneurysm series (table 1). The majority of patients were women $(70.4 \%)$ and the average age was $55.9 \pm 14.0$. About two-thirds of patients were diagnosed with subarachnoid hemorrhage (68.2\%). On average PC400 coils were used in the larger aneurysms $\left(218.9\right.$ vs $47.1 \mathrm{~mm}^{3}$, $\mathrm{p}<0.001)$ with greater dome/neck ratio ( 2.0 vs $1.6, \mathrm{p}=0.001)$, wider necks ( 3.0 vs $2.5 \mathrm{~mm}, \mathrm{p}=0.005)$ and in an aneurysm population with a higher percentage of high-risk aneurysms $(40.5 \%$ vs $15.2 \%, \mathrm{p}=0.001)$. When using the volume coil system we embolized fewer anterior communicating artery (ACOM) aneurysms (18.9\% vs $40.8 \%, \mathrm{p}=0.011)$ and more posterior communicating artery (PCOM) aneurysms $(32.4 \%$ vs $17.5 \%, \mathrm{p}=0.044)$ compared with the control group. Exclusion of aneurysms with a diameter $<7 \mathrm{~mm}$ increased the homogeneity of groups but we still report significant differences for aneurysm volume (246.7 vs $\left.154.3 \mathrm{~mm}^{3}, \mathrm{p}=0.001\right)$, maximum diameter (10.0 vs $8.8 \mathrm{~mm}$, $\mathrm{p}=0.023)$, and anterior communicating location $(16.1 \%$ vs $38.1 \%, p=0.026)$. In summary, even after excluding small aneurysms, volume coils were used in a potentially more difficult-to-treat group. Other baseline characteristics did not differ significantly between the groups.

\section{Procedure characteristics}

There was a difference in procedure time with increased procedure duration in the study group $(173.0 \mathrm{~min}$ vs $118.0 \mathrm{~min}$, $\mathrm{p}=0.032 ; \geq 7 \mathrm{~mm}: 179$ vs $112 \mathrm{~min}, \mathrm{p}=0.032$ ) (table 2). This difference became less prominent when mixed coil cases in the study group were excluded (123.5 vs $118.0 \mathrm{~min}, \mathrm{p}=0.454$; $\geq 7 \mathrm{~mm}$ : 161.5 vs $112.0 \mathrm{~min}, \mathrm{p}=0.238)$. We therefore believe that this observation may be due to a higher frequency of required catheter exchanges. In $29.7 \%$ of PC400 cases we additionally used standard diameter coils for neck finishing. However, these coils accounted for a very small volume fraction of the overall inserted coil mesh volume (11.5\% $0.4 \%)$. Aneurysms in the volume coil group were treated with fewer coils per cubic millimeter than those in the control group $(0.028$ vs $0.120, p<0.001)$. We did not observe any significant differences in either group for the frequency of stent or balloon assistance, which was around $15 \%$ each (all p $>0.5$ ). For wideneck aneurysms in our aneurysm population (neck width $\geq 4 \mathrm{~mm} ; \mathrm{n}=61$ ) we observed less stent assistance in the volume coil group than in the conventionally treated group $(16.7 \%$ vs $40.8 \%, \mathrm{p}=0.182$ ). However, this difference was not statistically significant. Overall we achieved higher packing density with the use of PC400 coils compared with the control group (43.2\% vs $34.4 \%, \mathrm{p}<0.001)$. This difference was also more pronounced when analyzing aneurysms $\geq 7 \mathrm{~mm} \quad(42.2 \%$ vs $27.8 \%$, 
Table 1 Baseline patient and aneurysm characteristics

\begin{tabular}{|c|c|c|c|c|c|c|}
\hline Characteristics & CC $(n=223)$ & VC $(n=37)$ & p Value & $C C \geq 7 \mathrm{~mm}(\mathrm{n}=84)$ & $\begin{array}{l}V C \geq 7 \mathrm{~mm} \\
(\mathrm{n}=31)\end{array}$ & p Value \\
\hline Age (years) & $55.29 \pm 13.84$ & $58.95 \pm 13.71$ & 0.140 & $57.2( \pm 13.2)$ & $58.4( \pm 14.1)$ & 0.674 \\
\hline Sex, female & $69.5 \%(155)$ & $75.7 \%(28)$ & 0.561 & $66.7 \%(56)$ & $77.4 \%(24)$ & 0.362 \\
\hline Ruptured & $66.4 \%(148)$ & $62.2 \%(23)$ & 0.709 & $67.9 \%(57)$ & $58.1 \%(18)$ & 0.380 \\
\hline \multicolumn{7}{|l|}{ Location } \\
\hline ICA & $13.5 \%(30)$ & $21.6 \%(8)$ & 0.210 & $11.9 \%(10)$ & $25.8 \%(8)$ & 0.085 \\
\hline MCA & $7.6 \%(17)$ & $8.1 \%(3)$ & 1.000 & $6.0 \%(5)$ & $9.7 \% \%(3)$ & 0.443 \\
\hline ACOM & $40.8 \%(91)$ & $18.9 \%(7)$ & $0.011^{*}$ & $38.1 \%(32)$ & $16.1(5)$ & $0.026^{*}$ \\
\hline $\mathrm{A} 1 / \mathrm{A} 2$ & $6.7 \%(15)$ & $0 \%(0)$ & 0.139 & $3.6 \%(3)$ & $0 \%(0)$ & 0.562 \\
\hline VA/PICA & $2.2 \%(5)$ & $2.7 \%(1)$ & 1.000 & $2.4 \%(2)$ & $0 \%(0)$ & 1.000 \\
\hline BA/AICA/SUCA & $9.9 \%(22)$ & $16.2 \%(6)$ & 0.254 & $15.5 \%(13)$ & $16.1(5)$ & 1.000 \\
\hline PCOM & $17.5 \%(39)$ & $32.4 \%(12)$ & $0.044^{*}$ & $21.4 \%(18)$ & $32.3 \%(10)$ & 0.233 \\
\hline $\mathrm{P} 1 / \mathrm{P} 2$ & $1.8 \%(4)$ & $0 \%(0)$ & 1.000 & $1.2 \%(1)$ & $0 \%(0)$ & 1.000 \\
\hline \multicolumn{7}{|l|}{ Aneurysm size } \\
\hline Small (<7 mm) & $62.3 \%(139)$ & $16.2 \%(6)$ & $<0.001 * *$ & - & - & \\
\hline Medium (7-12 mm) & $31.4 \%(70)$ & $59.5 \%(22)$ & $0.001^{* *}$ & $83.3 \%(70)$ & $71.0 \%(22)$ & 0.188 \\
\hline Large (>12 mm) & $6.3 \%(14)$ & $24.3 \%(9)$ & $0.002^{* *}$ & $16.7 \%(14)$ & $29.0 \%(9)$ & 0.188 \\
\hline Aneurysm volume $\left(\mathrm{mm}^{3}\right)$ & $47.1(16.0-116.7)$ & $218.9(114.5-462.8)$ & $<0.001 * *$ & 154.3 (89.8-241.6) & $246.7(182.2-508.3)$ & 0.001 ** \\
\hline Aneurysm max. diameter (mm) & $6.0(4.0-8.0)$ & $9.0(7.9-12.1)$ & $<0.001 * *$ & $8.8(7.9-10.0)$ & $10.0(8.0-12.8)$ & $0.023^{*}$ \\
\hline Neck width (mm) & $2.5(2.0-3.5)$ & $3.0(2.7-4.0)$ & $0.005^{* *}$ & $3.2(3.0-4.0)$ & $3.0(3.0-4.0)$ & 0.286 \\
\hline Dome/neck ratio & $1.6(1.3-2.1)$ & $2.0(1.6-3.1)$ & $0.001 * *$ & $1.9(1.6-2.5)$ & $2.3(1.6-3.1)$ & 0.094 \\
\hline High-risk aneurysms & $15.2 \%(34)$ & $40.5 \%(15)$ & $0.001^{* *}$ & $32.1 \%(27)$ & $45.2 \%(14)$ & 0.272 \\
\hline
\end{tabular}

$\mathrm{p}<0.001)$. There were fewer aneurysms with a packing density $<30 \%$ in the volume coil group $(10.8 \%$ vs $39.0 \%, p=0.001$; $\geq 7 \mathrm{~mm}: 9.7 \%$ vs $56.0 \%, \mathrm{p}<0.001$ ), while the portion of densely packed aneurysms $>40 \%$ was higher $(54.1 \%$ vs $30.9 \%$, $\mathrm{p}<0.008 ; \geq 7 \mathrm{~mm}: 54.8 \%$ vs $13.1 \%, \mathrm{p}<0.001)$. We also observed a previously reported general tendency towards lower packing densities in larger aneurysms. The downtrend was comparable in both groups but on a higher baseline level when using the PC400 coil (see figure 1). Although we did not find any significant difference in occlusion grade after procedure in both groups, there was a tendency towards more grade I occlusion grades in the PC400 group in medium and large aneurysms $(54.8 \%$ vs $38.1 \%, \mathrm{p}=0.138)$. Overall procedure-related complications $(10.8 \%$ vs $18.4 \%, \mathrm{p}=0.350 ; \geq 7 \mathrm{~mm}$ : $12.9 \%$ vs $19.0 \%$, $\mathrm{p}=0.583$ ) and detailed subgroup complication analysis did not differ significantly between the groups (all $p>0.25$ ). Two out of four complications in the volume group occurred in mixed-coil cases.

\section{Follow-up analysis}

Angiographic follow-up was available in $57.7 \%$ of all aneurysms (table 3). Median time to follow-up was in line with our institutional recommendation of a 6 month control $(6.0$ months; $\geq 7 \mathrm{~mm}$ : 6.0 months) and did not differ between the groups. We report a tendency towards more MRRC grade I $(61.9 \%$ vs $35.7 \%, \mathrm{p}=0.067)$, which did not reach statistical significance and fewer grade IIIa/b $(23.8 \%$ vs $52.3 \%, \mathrm{p}=0.035)$ on follow-up images in the study group when focusing on aneurysms $\geq 7 \mathrm{~mm}$. In this diameter subgroup we also noted less coil compaction $(23.8 \%$ vs $64.3 \%, \mathrm{p}=0.003)$ and less aneurysm recurrence $(14.3 \%$ vs $40.5 \%, \mathrm{p}=0.046)$ as well as a lower frequency of overall treatment failure $(16.1 \%$ vs $38.1 \%, \mathrm{p}=0.026)$ when using volume coils (see figure 2 for an illustrative case). In all recurrent aneurysms, coil compaction was reported (33/33), whereas only $58.9 \%(33 / 56)$ of coil compaction cases resulted in aneurysm recurrence. This is mainly explained by the fact that recurrence was not defined as any worsening compared with postprocedural occlusion grade, but as an occlusion grade of IIIb/IIIa on follow-up with better occlusion rates on postinterventional control images. So cases with occlusion grade worsening from MRRC grade I to MRRC grade II and cases, both with postprocedural and follow-up MRRC grade IIIb, did not count as aneurysm recurrence, but we report coil compaction. We also did not observe any statistically significant difference of compaction frequency within the packing density groups when comparing volume coils with those of the control group (20-30\%, $\mathrm{p}=1.000,30-40 \%, \mathrm{p}=0.694,>40 \%, \mathrm{p}=0.694)$, which suggests that outcome differences between the groups are mainly due to a greater frequency of densely packed aneurysms in the volume coil group, rather than other material property differences. The best cut-off packing density value for overall treatment failure in all 260 analyzed aneurysms was determined as 25.5\% (area under the curve $=0.702$; highest Youden index $=0.34$ ).

\section{Catheter-placement difficulties}

We also observed six cases with an intention-to-treat with PC400 coils, but applied coiling technique was changed during procedure (table 4). All six aneurysms were in the anterior circulation while four of the six had a distal location (two ACOM, one M1, one A1). In five of the six cases it was possible to safely change the catheter type to a smaller one (Echelon 10, Covidien, Irvine, California, USA or Excelsior SL10, Stryker, Fremont, California, USA) and successfully finish endovascular treatment using standard diameter coils (see figure 3 for an 
Table 2 Procedure information

\begin{tabular}{|c|c|c|c|c|c|c|}
\hline & $C C(n=223)$ & VC $(n=37)$ & p Value & $\mathrm{CC} \geq 7 \mathrm{~mm}(\mathrm{n}=84)$ & $\begin{array}{l}V C \geq 7 \mathrm{~mm} \\
(\mathrm{n}=31)\end{array}$ & p Value \\
\hline Procedure time (min) & $139.2 / 118.0(17-464)$ & $163.2 / 173.0(52-366)$ & $0.032^{*}$ & $139.8 / 112.0(41-350)$ & $167.2 / 179.0(52-366)$ & $0.032^{*}$ \\
\hline Procedure time w/o mixed coil cases (min) & $139.2 / 118.0(17-464)$ & $151.3 / 123.5(52-366)$ & 0.454 & $139.8 / 112.0(41-350)$ & $162.4 / 161.5(52-366)$ & 0.238 \\
\hline PC400/conventional mixed & - & $29.7 \%(11)$ & & - & $29.0 \%(9)$ & - \\
\hline \multicolumn{7}{|l|}{ Technique } \\
\hline Stand-alone coiling & $66.4 \%(148)$ & $70.3 \%(26)$ & 0.709 & $66.7 \%(56)$ & $71.0 \%(22)$ & 0.823 \\
\hline Stent-assisted coiling & $13.9 \%(31)$ & $13.5 \%(5)$ & 1.000 & $19.0 \%(16)$ & $12.9 \%(4)$ & 0.583 \\
\hline Balloon-assisted coiling & $18.8 \%(42)$ & $13.5 \%(5)$ & 0.644 & $14.3 \%(12)$ & $16.1 \%(5)$ & 0.774 \\
\hline Packing density & $34.4 \pm 14.9$ & $43.2 \pm 12.9$ & $<0.001$ ** & $27.8 \pm 10.7$ & $42.2 \pm 10.0$ & $<0.001$ * * \\
\hline \multicolumn{7}{|l|}{ Packing density groups } \\
\hline $0-10 \%$ & $2.7 \%(6)$ & $0(0 \%)$ & 0.599 & $4.8 \%(4)$ & $0 \%(0)$ & 0.573 \\
\hline $10-20 \%$ & $11.7 \%(26)$ & $0(0 \%)$ & $0.033^{*}$ & $16.7 \%(14)$ & $0 \%(0)$ & $0.011^{*}$ \\
\hline $20-30 \%$ & $24.7 \%(55)$ & $10.8 \%(4)$ & 0.088 & $34.5 \%(29)$ & $9.7 \%(3)$ & $0.009 * *$ \\
\hline $30-40 \%$ & $30.0 \%(67)$ & $35.1 \%(13)$ & 0.566 & $31.0 \%(26)$ & $35.5 \%(11)$ & 0.658 \\
\hline$>40 \%$ & $30.9 \%(69)$ & $54.1 \%(20)$ & $0.008^{*}$ & $13.1 \%(11)$ & $54.8 \%(17)$ & $<0.001^{* *}$ \\
\hline \multicolumn{7}{|l|}{ MRRC grade after procedure } \\
\hline I & $52.0 \%(116)$ & $54.1 \%(20)$ & 0.860 & $38.1 \%(32)$ & $54.8 \%(17)$ & 0.138 \\
\hline II & $16.6 \%(37)$ & $16.2 \%(6)$ & 1.000 & $21.4 \%(18)$ & $9.7 \%(3)$ & 0.182 \\
\hline IIla & $19.3 \%(43)$ & $21.6 \%(8)$ & 0.823 & $22.6 \%(19)$ & $25.8 \%(8)$ & 0.805 \\
\hline IIIb & $12.1 \%(27)$ & $8.1 \%(3)$ & 0.590 & $17.9 \%(15)$ & $9.7 \%(3)$ & 0.391 \\
\hline \multicolumn{7}{|l|}{ Procedure-related complication } \\
\hline Total & $18.4 \%(41)$ & $10.8 \%(4)$ & 0.350 & $19.0 \%(16)$ & $12.9 \%(4)$ & 0.583 \\
\hline Aneurysm rupture & $2.7 \%(6)$ & $2.7 \%(1)$ & 1.000 & $0 \%(0)$ & $3.2 \%(1)$ & 0.270 \\
\hline Coil malposition & $2.7 \%(6)$ & $0 \%(0)$ & 1.000 & $6.0 \%(5)$ & $3.2 \%(1)$ & 1.000 \\
\hline Vasospasm & $4.0 \%(9)$ & $0 \%(0)$ & 0.367 & $1.2 \%(1)$ & $0 \%(0)$ & 1.000 \\
\hline Thrombosis & $7.6 \%(17)$ & $5.4 \%(2)$ & 1.000 & $8.3 \%(7)$ & $6.5 \%(2)$ & 1.000 \\
\hline Parent artery occlusion & $0.4 \%(1)$ & $0 \%(0)$ & 1.000 & $0 \%(0)$ & $0 \%(0)$ & - \\
\hline Embolic event & $1.3 \%(3)$ & $0 \%(0)$ & 1.000 & $3.6 \%(3)$ & $0 \%(0)$ & 0.562 \\
\hline
\end{tabular}

illustrative case). In one case catheter placement was insufficient even after changing the catheter to a smaller one, and here surgical clipping was performed.

\section{DISCUSSION}

To the best of our knowledge, six case reviews have evaluated the properties, efficacy, and safety of the PC400 coil system. ${ }^{19-21}$ 28-30 The largest PC400 case series $(\mathrm{n}=76)$ by Mascitelli et $a l^{28}$ reported higher packing density $(31.7 \%$ vs $24.8 \%, \mathrm{p}<0.05)$, shorter procedure time ( 48 vs $64 \mathrm{~min}, \mathrm{p}<0.05$ ), and a smaller number of coils used for each aneurysm ( 3.53 vs $5.44, p=0.002$ ), but no difference in the frequency rates of stent and balloon assists, in comparison with conventional treatment. They report a frequency of Raymond-Roy occlusion classification grade III aneurysms after coil placement of $71.1 \%$ in the study (PC400 coils) group and $38.2 \%$ in their control group using conventional coils $(\mathrm{p}<0.001)$. Contrary to their postprocedural findings they report comparable grade III occlusion rate on follow-up images (20.5\% vs $22.8 \%, \mathrm{p}=0.837$ ), which is explained by a higher rate of improved aneurysms in the PC400-treated group $(51.3 \%$ vs $27.7 \%, \mathrm{p}=0.009)$. Milburn et al ${ }^{20}$ compared 18 aneurysms treated with the PC400 system with a control group that underwent endovascular occlusion with Orbit and Galaxy coils. They also reported higher packing densities $(33.7 \%$ vs $24.4 \%$, $\mathrm{p}<0.001$ ), a smaller number of coils per cubic millimeter aneurysm volume $(0.026$ vs $0.114, \mathrm{p}<0.001)$, and an associated improved cost efficiency. Woo et $a l^{29}$ reported an average packing density of $35.7 \%$ without observing any device-related adverse events when using PC400 coils in a small study group $(n=7)$. Baxter and Quarfordt ${ }^{30}$ reported a packing density of $48.9 \%$ and the use of fewer coils for each aneurysm, with their follow-up data suggesting durable occlusion rates when using the PC400 system in 34 small, medium, and large aneurysms. Villwock et $a l^{21}$ found that wide-neck aneurysms treated with either PC400 or conventional coils required a lower frequency of stent assistance when using the volume coil system.

In this study we have shown that endovascular aneurysm occlusion using the PC400 in aneurysms $\geq 7 \mathrm{~mm}$ results in the use of fewer coils per volume unit, a comparable incidence of adverse events, increased packing density, more completely occluded aneurysms, less coil compaction, less aneurysm recurrence, and less overall treatment failure compared with standard coil treatment. We also found six intention-to-treat cases, in which it was not possible to use the PC400 system owing to the required use of a catheter with a larger diameter.

We report higher mean packing densities when PC400 coils are used compared with previously published data. In the PC400 group our average packing density was $43.2 \%$, while we calculated the average of previously published data to be $36.3 \%$ $(n=135)$. Our rate of postinterventional fully occluded aneurysms was also much higher than for the study group of Mascitelli et al (54.1\% vs $13.2 \%)$. Both might be explained by overall more aggressive treatment or a higher rate of additional use of conventional coils for neck finishing. On follow-up we 


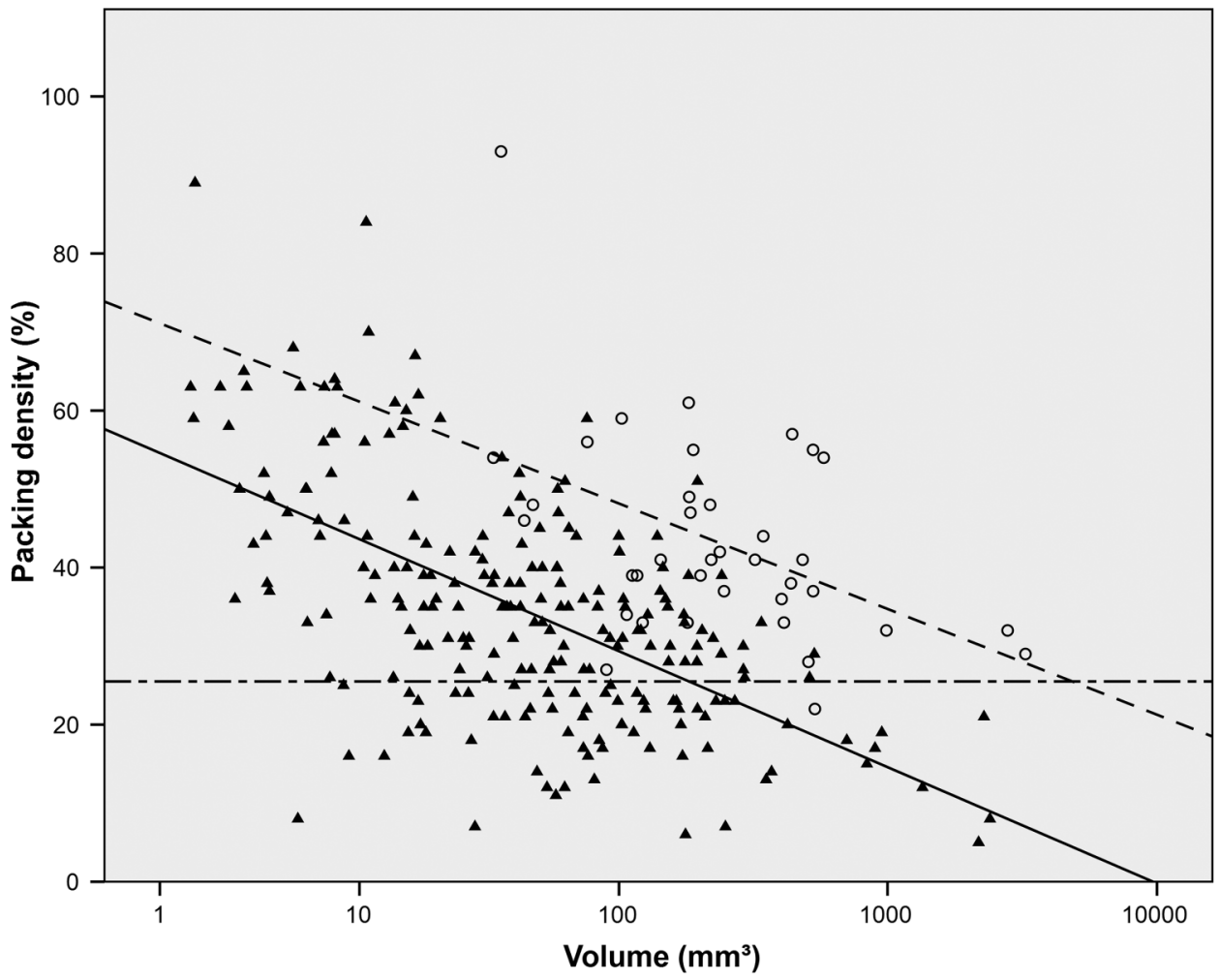

Figure 1 Volume and packing density of aneurysms treated with either Penumbra Coil 400 (PC400) coils or standard diameter coils. $\mathbf{\Delta}$, standard diameter coils with linear regression model (-); $\bigcirc$, PC400 coils with linear regression model $(----) ; x$ axis, logarithmic volume in mm ${ }^{3}$, y axis packing density in \%; y axis reference line (- - - - ), 25.5\% indicates calculated best cut-off packing density from our study data.

report $28.0 \%$ grade IIIa/IIIb aneurysms, which is slightly higher than the $20.5 \%$ reported by Mascitelli et al. On the other hand, we observed more grade I (60.0\%) aneurysms than in their study group (40.6\%). The overall higher recurrence rate might be explained by the overall risk factor distribution described below. Compared with our PC400-treated aneurysms, their study group consisted of aneurysms with smaller volume (median 110.8 vs $218.9 \mathrm{~mm}^{3}$ ), smaller aneurysm diameter (mean 6.5 vs $10.2 \mathrm{~mm}$ ), and a lower percentage of ruptured aneurysms (44.2\% vs $62.2 \%)$. Owing to these attributes, their patients had a lower risk of increased recurrence rates.

Contrary to previous studies we did not note a shortening of procedure time but a tendency towards an increased procedure duration in the volume coil group as well as an overall prolonged intervention time compared with other reports. Those findings might be explained by the use of different procedure time definitions. Previous studies defined procedure time as the time between the first working view image and the first control image, whereas we set procedure time as the difference between the first angiographic image and the first control image. Taking into account that one has to use a larger catheter we hypothesize that a suggested shorter coil insertion time, might be compensated by a longer probing time and a more difficult catheter placement. In addition, as previously mentioned, we report a higher frequency of neck finishing with standard coils in the PC400 group, thus requiring catheter exchange, which may take

Table 3 Follow-up outcome

\begin{tabular}{|c|c|c|c|c|c|c|}
\hline & $C C(n=223)$ & VC $(n=37)$ & $\mathrm{p}$ Value & $\mathrm{CC} \geq 7 \mathrm{~mm}(\mathrm{n}=84)$ & $V C \geq 7 \mathrm{~mm}(n=31)$ & p Value \\
\hline FU available & $56.1 \%(125)$ & $67.6 \%(25)$ & 0.212 & $50.0 \%(42)$ & $67.7 \%(21)$ & 0.097 \\
\hline Median time to FU & $5.9(4.4-6.8)$ & $6(4.7-7.1)$ & 0.572 & $6.0(5.0-7.3)$ & $5.9(5.3-6.8)$ & 0.586 \\
\hline \multicolumn{7}{|l|}{ MRRC grade at FU } \\
\hline 1 & $55.2 \%(69 / 125)$ & $60.0 \%(15 / 25)$ & 0.826 & $35.7 \%(15 / 42)$ & $61.9 \%(13 / 21)$ & 0.063 \\
\hline ॥ & $12.0 \%(15 / 125)$ & $12.0 \%(3 / 25)$ & 1.000 & $11.9 \%(5 / 42)$ & $14.3 \%(3 / 21)$ & 1.000 \\
\hline Illa & $4.0 \%(5 / 125)$ & $4.0 \%(1 / 25)$ & 1.000 & $2.3 \%(1 / 43)$ & $4.8 \%(1 / 21)$ & 1.000 \\
\hline Illb & $28.8 \%(36 / 125)$ & $24.0 \%(6 / 25)$ & 0.808 & $50.0 \%(21 / 42)$ & $19.0 \%(4 / 21)$ & $0.028^{*}$ \\
\hline IIla/lllb & $33.6 \%(42 / 125)$ & $28.0 \%(7 / 25)$ & 0.815 & $52.4 \%(22 / 42)$ & $23.8 \%(5 / 21)$ & $0.035^{*}$ \\
\hline Coil compaction & $39.2 \%(49 / 125)$ & $28.0 \%(7 / 25)$ & 0.368 & $64.3 \%(27 / 42)$ & $23.8 \%(5 / 21)$ & $0.003^{* *}$ \\
\hline Aneurysm recurrence & $22.4(28 / 125)$ & $20.0 \%(5 / 25)$ & 1.000 & $40.5 \%(17 / 42)$ & $14.3 \%(3 / 21)$ & $0.046^{*}$ \\
\hline Overall treatment failure & $25.6 \%(57 / 223)$ & $18.9 \%(7 / 37)$ & 0.420 & $38.1 \%(32 / 84)$ & $16.1 \%(5 / 31)$ & $0.026^{*}$ \\
\hline
\end{tabular}

${ }^{*} \mathrm{p}<0.05,{ }^{* *} \mathrm{p}<0.01$; normally distributed variables shown as mean $\pm S D$, non-normally distributed as median (IQR).

CC, conventional coils; FU, follow-up; MRRC, modified Raymond-Roy occlusion classification; VC, volume coils. 


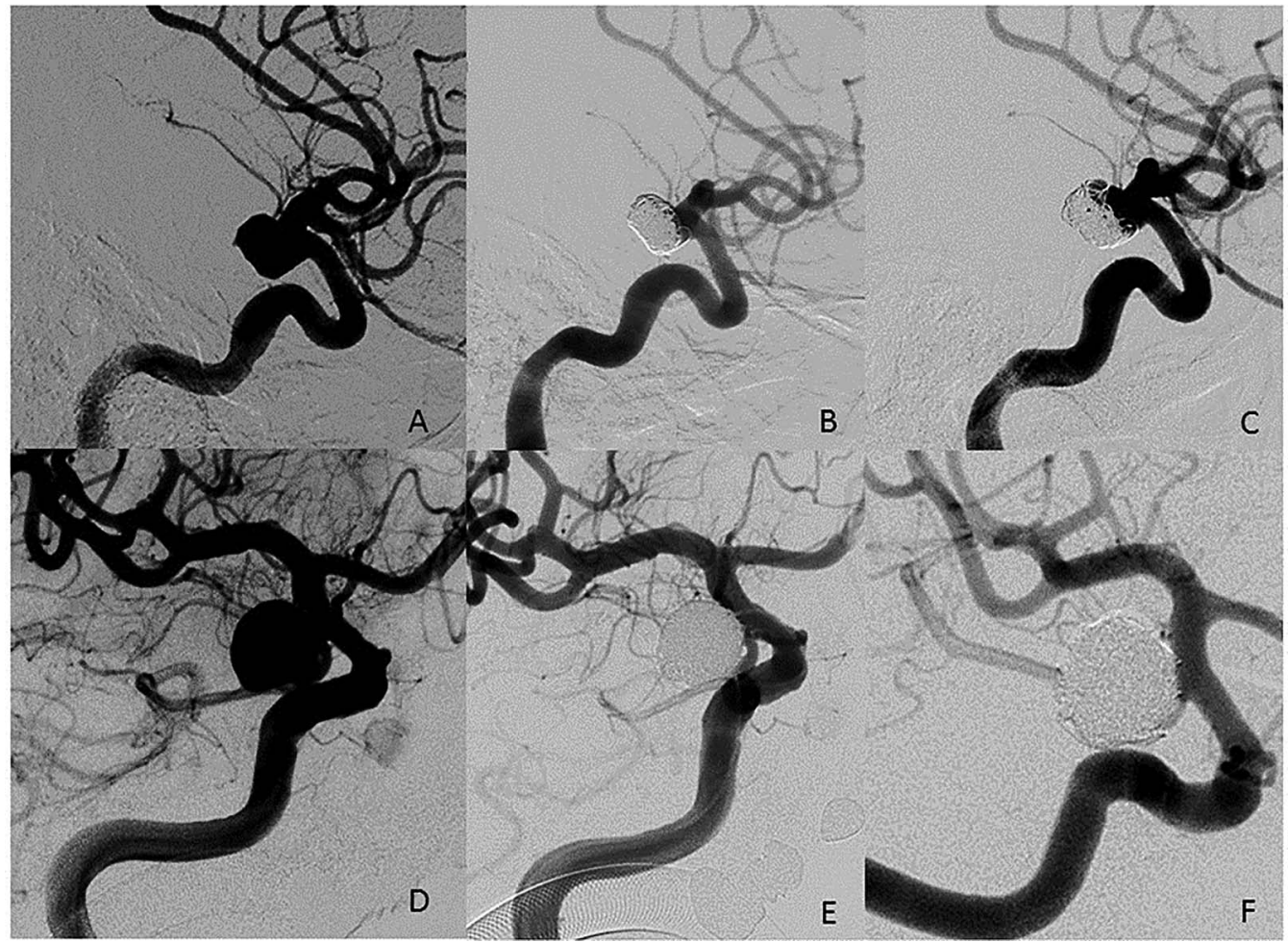

Figure 2 Illustrative case comparison of two large posterior communicating artery (PCOM) aneurysms. (A and D) Aneurysm before coil embolization; ( $B$ and $E)$ postprocedural control image; ( $C$ and $F$ ) angiographic follow-up control. ( $A-C)$ PCOM aneurysm treated with conventional coils; volume $2907 \mathrm{~mm}^{3}$; maximum diameter $12 \mathrm{~mm}$; neck width $4.0 \mathrm{~mm}$ : packing density $30 \%$; initial modified Raymond-Roy occlusion classification (MRRC) grade II; follow-up MRRC grade (6.2 months) grade Illb. (D-F) PCOM aneurysm treated with volume coils; volume $579.0 \mathrm{~mm}^{3}$; maximum diameter $12.8 \mathrm{~mm}$; neck width $2.5 \mathrm{~mm}$; packing density 54\%; initial MRRC grade I; follow-up MRRC grade (5.1 months) I.

several minutes. We are aware that a long procedure duration is generally associated with an increased complication rate, but we did not observe more procedure-related adverse events in the study group. ${ }^{31}$

We also reported a lower percentage of treated ACOM aneurysms when using the volume coil system, which is well in line with aneurysm location characteristics treated with PC400 coils

Table 4 Probing or catheter placement failure when using the PC400 system

\begin{tabular}{|c|c|c|c|c|}
\hline $\begin{array}{l}\text { Case } \\
\text { No }\end{array}$ & Location & $\begin{array}{l}\text { PC400 } \\
\text { catheter }\end{array}$ & $\begin{array}{l}\text { Catheter change } \\
\text { to }\end{array}$ & $\begin{array}{l}\text { Successfully } \\
\text { treated by }\end{array}$ \\
\hline 1 & ICA & $\begin{array}{l}90^{\circ} \text { tip PX } \\
\text { Slim }\end{array}$ & Echelon 10 & Coiling \\
\hline 2 & $\mathrm{ACOM}$ & $\begin{array}{l}45^{\circ} \text { tip PX } \\
\text { Slim }\end{array}$ & Excelsior SL10 & Coiling \\
\hline 3 & M1 & $\begin{array}{l}45^{\circ} \text { tip PX } \\
\text { Slim }\end{array}$ & $\begin{array}{l}\text { Echelon } 10,90^{\circ} \\
\text { tip shape }\end{array}$ & Coiling \\
\hline 4 & $\mathrm{~A} 1$ & $\begin{array}{l}45^{\circ} \text { tip PX } \\
\text { Slim }\end{array}$ & Excelsior SL10 & Coiling \\
\hline 5 & PCOM & $\begin{array}{l}45^{\circ} \text { tip PX } \\
\text { Slim }\end{array}$ & Excelsior SL10 & Coiling \\
\hline 6 & ACOM & $45^{\circ} \mathrm{PX} 400$ & $\begin{array}{l}\text { Echelon } 14,45^{\circ} \\
\text { tip shape }\end{array}$ & Clipping \\
\hline
\end{tabular}

A1, segment 1 of the anterior cerebral artery; ACOM, anterior communicating artery; Echelon 10, Covidien, Irvine, California, USA; Excelsior SL10, Stryker, Fremont, California, USA; ICA, internal carotid artery; M1, segment 1 of the middle cerebral artery; PC400, Penumbra Coil 400, Penumbra, Inc., Alameda, California, USA; PCOM, posterior communicating artery; PX400/ PX Slim, Penumbra, Inc., Alameda, California, USA. reported by Mascitelli et al. ${ }^{28}$ ACOM aneurysms often exhibit complex parent vessel relationships and are frequently associated with anomalies of the ACOM. They are associated with a smaller A1-A2 angle junction and hypoplastic or aplastic A1 segments. ${ }^{32}$ Considering the tortuosity and length, it is more complicated to control and safely place coils and possible assistance devices. We think that those factors led interventionalists to favor smaller microcatheters and thereby the use of standard diameter coils when treating ACOM aneurysms.

A further observation in the study of Mascitelli $e t \mathrm{al}^{28}$ and in our study is an overall increased frequency of PCOM aneurysms treated with the PC400. Usually the fundus of PCOM aneurysms is in line with the communicating segment of the internal carotid artery (ICA) and does not require a second catheter turn shortly after the cavernous segment of the ICA. Additionally, the incidence of unilateral or bilateral fetal PCOM variants is around $25 \%,{ }^{33-36}$ and this variant often results in a vessel anatomy that is relatively conducive to catheter placement. For these reasons we believe that interventionalists felt comfortable using the larger catheter, which is necessary for implementing volume coils, when encountering aneurysms of the PCOM. Overall, we also believe that localization bias should not affect outcome parameters as we are not aware of any study which has shown a statistically significant association between localization and angiographic aneurysm recurrence after coil embolization.

As mentioned above we observed six cases in which it was not possible to use the PC400 coil system. Five of the six aneurysms in the intention-to-treat group could be safely occluded using conventional embolization techniques. This affects the relative superiority of the PC400 system by demonstrating device-specific technical limitations. We suggest that the PC400 


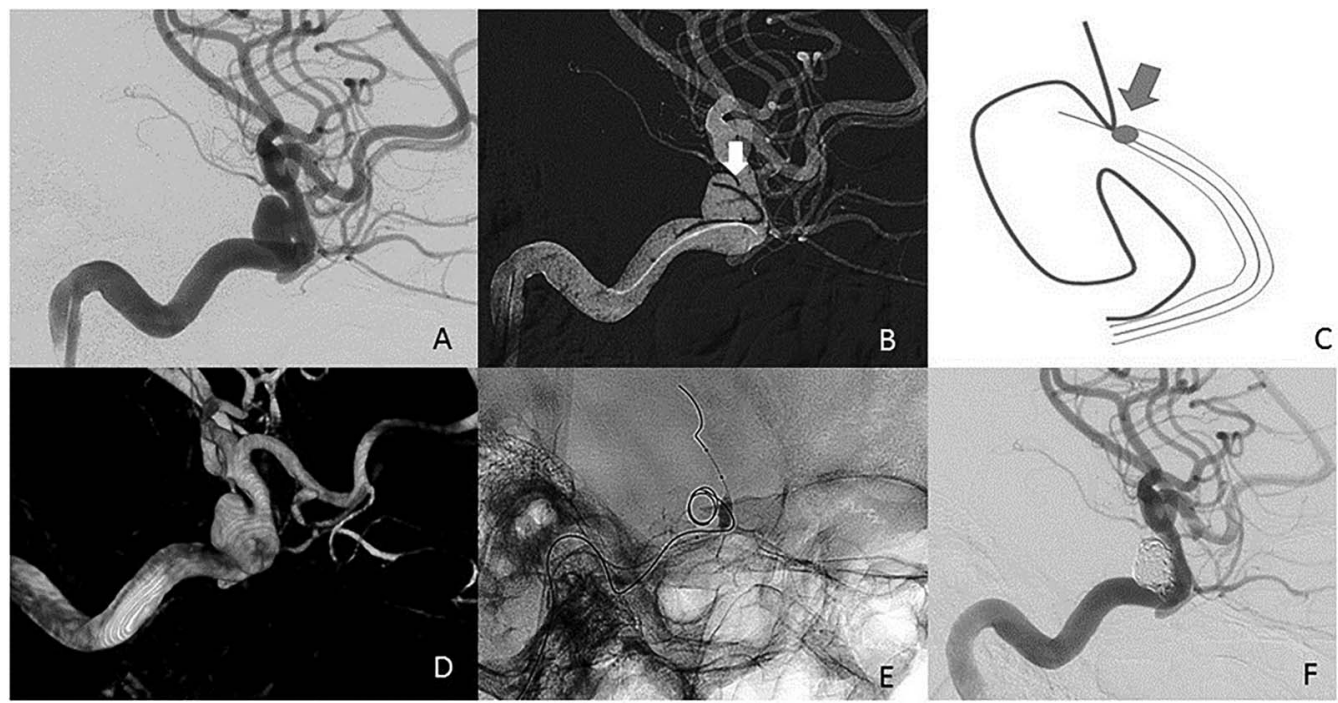

Figure 3 Illustrative case of catheter management in an internal carotid artery (ICA) aneurysm. (A and D) ICA aneurysm before coil embolization. (B) Unsuccessful catheter placement of the PX Slim owing to steep ICA/aneurysm curve. (C) Owing to the radial bending force caused by the proximal steep vessel curve, the guide wire was pressed against the cranial aneurysm neck wall. It was not possible to pass the narrow neck passage which occurred with the PX Slim (Penumbra, Inc, Alameda, California, USA) catheter (arrow). (E) Successful balloon-assisted catheter (Excelsior SL10, Stryker, Fremont, California, USA) placement. (F) Successful occlusion using standard diameter coils (modified Raymond-Roy occlusion classification grade I).

system is not ideal for treating small and distal aneurysms, mainly because of the need for a bigger and more rigid catheter. We also advocate a change in the technical treatment regimen to a smaller catheter during intervention whenever placement of the PX Slim is not feasible.

Several studies suggested that increased packing density might prevent aneurysm recanalization by decreasing coil compaction

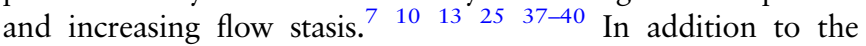
effect of the overall packing density itself, there is also some evidence that the homogeneity of coil distribution is an important cofactor. $^{41}{ }^{42}$ Although Goddard et al found that packing density was predicative of recurrence only for medium and large aneurysms, there is still debate about which subgroups are most influenced by packing density variations and their effect on aneurysm recanalization. ${ }^{43}$ A variety of best cut-off values for recurrence and treatment failure have been proposed. Kawanabe et $a l^{10}$ and Leng et $a l^{13}$ both found a packing density of $20 \%$ to be the best cut-off point in preventing recurrence. Sluzewski et $a l^{7}$ reported no coil compaction when an aneurysm is packed $\geq 25 \%$. A recent study by Mascitelli et $a l^{25}$ proposed a packing density of $31 \%$ as the best-fit cut-off point. In our study we identified $25.5 \%$ to be the most significant cut-off point for overall treatment failure, which is consistent with the results of other institutions. It should be kept in mind that our results are based on a retrospective study and that clinical outcome parameters are not addressed.

The decision about which coil type to use for a given patient was made by interventionalists based on the aneurysm characteristics rather than randomly, which might have resulted in a selection bias and unobserved heterogeneity in the sample. Most of the PC400 aneurysms were treated more recently than those aneurysms treated with standard diameter coils, which might have affected the results owing to an overall skill progression.

\section{CONCLUSION}

The results of our monocentric retrospective case series study on the administration of the PC400 volume coil system as opposed to conventional diameter coils suggest that the PC400 volume coil system is safe and effective in treating intracranial aneurysms. Despite having been applied in a potentially more difficult-to-treat group, the use of PC400 was associated with less coil compaction and aneurysm recurrence in aneurysms $\geq 7 \mathrm{~mm}$, probably owing to the higher packing density achieved with PC400 coils.

Contributors JK analyzed and interpreted the data, performed the statistical analysis, and drafted the initial manuscript. TH analyzed and interpreted the data and made critical revisions to the manuscript. SP, FD, CZ and TB-B designed and conceived the research and made critical revisions to the manuscript. ES, CM-L and $\mathrm{BH}$ acquired the data and performed the statistical analysis. All authors participated in the review, editing, and final approval of the manuscript. All authors are guarantors of the integrity of the entire study.

Competing interests SP and TB-B are investigators in the ACE study (NCT01465841)

Ethics approval We declare that all human studies have been approved by the ethics committee of the TUM School of Medicine and have therefore been performed in accordance with the ethical standards laid down in the 1964 Declaration of Helsinki and its later amendments.

Provenance and peer review Not commissioned; externally peer reviewed.

Data sharing statement Some data generated during the project will be made freely available via http://datadryad.org/. DOls to these data will be provided.

\section{REFERENCES}

1 Brilstra EH, Rinkel GJ, van der Graaf $Y$, et al. Treatment of intracranial aneurysms by embolization with coils: a systematic review. Stroke 1999;30:470-6.

2 Molyneux A, Kerr R, International Subarachnoid Aneurysm Trial Collaborative Group, et al. International Subarachnoid Aneurysm Trial (ISAT) of neurosurgical clipping versus endovascular coiling in 2143 patients with ruptured intracranial aneurysms: a randomized trial. J Stroke Cerebrovasc Dis 2002;11:304-14.

3 Molyneux AJ, Birks J, Clarke A, et al. The durability of endovascular coiling versus neurosurgical clipping of ruptured cerebral aneurysms: 18 year follow-up of the UK cohort of the International Subarachnoid Aneurysm Trial (ISAT). Lancet 2015;385:691-7.

4 Molyneux AJ, Kerr RS, Birks J, et al. Risk of recurrent subarachnoid haemorrhage, death, or dependence and standardised mortality ratios after clipping or coiling of an intracranial aneurysm in the International Subarachnoid Aneurysm Trial (ISAT): long-term follow-up. Lancet Neurol 2009;8:427-33.

5 Molyneux AJ, Kerr RS, Yu LM, et al. International subarachnoid aneurysm trial (ISAT) of neurosurgical clipping versus endovascular coiling in 2143 patients with 
ruptured intracranial aneurysms: a randomised comparison of effects on survival, dependency, seizures, rebleeding, subgroups, and aneurysm occlusion. Lancet 2005;366:809-17.

6 Sluzewski M, van Rooij WJ, Rinkel GJ, et al. Endovascular treatment of ruptured intracranial aneurysms with detachable coils: long-term clinical and serial angiographic results. Radiology 2003;227:720-4.

7 Sluzewski M, van Rooij WJ, Slob MJ, et al. Relation between aneurysm volume, packing, and compaction in 145 cerebral aneurysms treated with coils. Radiology 2004;231:653-8

8 Raymond J, Guilbert F, Weill A, et al. Long-term angiographic recurrences after selective endovascular treatment of aneurysms with detachable coils. Stroke 2003:34:1398-403.

9 Ferns SP, Sprengers ME, van Rooij WJ, et al. Coiling of intracranial aneurysms: a systematic review on initial occlusion and reopening and retreatment rates. Stroke 2009;40:e523-9.

10 Kawanabe Y, Sadato A, Taki W, et al. Endovascular occlusion of intracranial aneurysms with Guglielmi detachable coils: correlation between coil packing density and coil compaction. Acta Neurochir (Wien) 2001;143:451-5.

11 Tamatani $\mathrm{S}$, Ito $\mathrm{Y}$, Abe $\mathrm{H}$, et al. Evaluation of the stability of aneurysms after embolization using detachable coils: correlation between stability of aneurysms and embolized volume of aneurysms. AJNR Am J Neuroradiol 2002;23:762-7.

12 Byrne JV, Sohn MJ, Molyneux AJ, et al. Five-year experience in using coil embolization for ruptured intracranial aneurysms: outcomes and incidence of late rebleeding. J Neurosurg 1999;90:656-63.

13 Leng $B$, Zheng $Y$, Ren J, et al. Endovascular treatment of intracranial aneurysms with detachable coils: correlation between aneurysm volume, packing, and angiographic recurrence. J Neurointerv Surg 2014;6:595-9.

14 Slob MJ, van Rooij WJ, Sluzewski M. Influence of coil thickness on packing, re-opening and retreatment of intracranial aneurysms: a comparative study between two types of coils. Neurol Res 2005;27(Suppl 1):S116-19.

15 Slob MJ, van Rooij WJ, Sluzewski M. Coil thickness and packing of cerebral aneurysms: a comparative study of two types of coils. AJNR Am J Neuroradiol 2005;26:901-3.

16 Wakhloo AK, Gounis MJ, Sandhu JS, et al. Complex-shaped platinum coils for brain aneurysms: higher packing density, improved biomechanical stability, and midterm angiographic outcome. AJNR Am J Neuroradiol 2007;28:1395-400.

17 Eddleman CS, Welch BG, Vance AZ, et al. Endovascular coils: properties, technical complications and salvage techniques. J Neurointerv Surg 2013;5:104-9.

18 Mascitelli JR, Oermann EK, De Leacy RA, et al. Angiographic outcome of intracranial aneurysms with neck remnant following coil embolization. J Neurointerv Surg 2015;7:484-9.

19 Mascitelli JR, Polykarpou MF, Patel AA, et al. Initial experience with Penumbra coil 400 versus standard coils in embolization of cerebral aneurysms: a retrospective review. J Neurointerv Surg 2013;5:573-6.

20 Milburn J, Pansara AL, Vidal G, et al. Initial experience using the Penumbra coil 400: comparison of aneurysm packing, cost effectiveness, and coil efficiency. J Neurointerv Surg 2014;6:121-4.

21 Villwock MR, Singla A, Padalino DJ, et al. The penumbra coil 400 system for treatment of wide-necked intracranial aneurysms: initial single-center experience. Acta Neurochir Suppl 2015;120:191-5.

22 Woodward K, Forsberg DA. AngioSuite: an accurate method to calculate aneurysm volumes and packing densities. J Neurointerv Surg 2013;5 (Suppl 3):iii28-32.

23 Wiebers DO, Whisnant JP, Huston J III, et al Unruptured intracranial aneurysms: natural history, clinical outcome, and risks of surgical and endovascular treatment. Lancet 2003;362:103-10.

24 Mascitelli JR, Moyle H, Oermann EK, et al. An update to the Raymond-Roy Occlusion Classification of intracranial aneurysms treated with coil embolization. J Neurointerv Surg 2015;7:496-502.
25 Mascitelli JR, Oermann EK, De Leacy RA, et al. Predictors of treatment failure following coil embolization of intracranial aneurysms. J Clin Neurosci 2015;22:1275-81.

26 Ries T, Siemonsen S, Thomalla G, et al. Long-term follow-up of cerebral aneurysms after endovascular therapy prediction and outcome of retreatment. AJNR Am J Neuroradiol 2007;28:1755-61.

27 Vanzin JR, Mounayer C, Abud DG, et al. Angiographic results in intracranial aneurysms treated with inert platinum coils. Interv Neuroradiol 2012;18:391-400.

28 Mascitelli JR, Patel AB, Polykarpou MF, et al. Analysis of early angiographic outcome using unique large diameter coils in comparison with standard coils in the embolization of cerebral aneurysms: a retrospective review. J Neurointerv Surg 2015;7:126-30.

29 Woo H, Fiorella D, Han P. P-011 Preliminary results with a new volumetric-filling platinum coil, the penumbra coil 400. J Neurointerv Surg 2011;3(Suppl 1):A20.

30 Baxter B, Quarfordt S. P-028 Clinical experience and lessons learned with the penumbra PC 400 large volume coil: improving the treatment of both large and small aneurysms. J Neurointerv Surg 2012;4(Suppl 1):A35. 10.1136/ neurintsurg-2012-010455b.28

31 Willinsky RA, Taylor SM, TerBrugge K, et al. Neurologic complications of cerebral angiography: prospective analysis of 2,899 procedures and review of the literature. Radiology 2003;227:522-8.

32 Kasuya $H$, Shimizu T, Nakaya $K$, et al. Angles between $A 1$ and $A 2$ segments of the anterior cerebral artery visualized by three-dimensional computed tomographic angiography and association of anterior communicating artery aneurysms. Neurosurgery 1999;45:89-93; discussion 93-4.

33 Horikoshi T, Akiyama I, Yamagata Z, et al. Magnetic resonance angiographic evidence of sex-linked variations in the circle of willis and the occurrence of cerebral aneurysms. J Neurosurg 2002;96:697-703.

34 Bisaria KK. Anomalies of the posterior communicating artery and their potential clinical significance. J Neurosurg 1984;60:572-6.

35 Jongen JC, Franke CL, Ramos LM, et al. Direction of flow in posterior communicating artery on magnetic resonance angiography in patients with occipital lobe infarcts. Stroke 2004;35:104-8.

36 Rhoton AL, Jr. The supratentorial arteries. Neurosurgery 2002;51(4 Suppl): S53-120.

37 Grunwald IQ, Balami JS, Weber D, et al. Different factors influence recanalisation rate after coiling in ruptured and unruptured intracranial aneurysms. CNS Neurol Disord Drug Targets 2013;12:228-32.

38 Babiker MH, Gonzalez LF, Albuquerque F, et al. Quantitative effects of coil packing density on cerebral aneurysm fluid dynamics: an in vitro steady flow study. Ann Biomed Eng 2010;38:2293-301.

39 Otani T, Nakamura M, Fujinaka T, et al. Computational fluid dynamics of blood flow in coil-embolized aneurysms: effect of packing density on flow stagnation in an idealized geometry. Med Biol Eng Comput 2013;51:901-10.

40 Morales HG, Kim M, Vivas EE, et al. How do coil configuration and packing density influence intra-aneurysmal hemodynamics? AJNR Am J Neuroradiol 2011:32:1935-41.

41 Chueh J, Vedantham S, Puri A, et al. E-060 effect of packing density and coil uniformity on coil permeability. J Neurointerv Surg 2014; 6(Suppl 1):A66-7.

42 Chueh JY, Vedantham S, Wakhloo AK, et al. Aneurysm permeability following coil embolization: packing density and coil distribution. J Neurointerv Surg 2015;7:676-81.

43 Goddard JK, Moran CJ, Cross DT III, et al Absent relationship between the coil-embolization ratio in small aneurysms treated with a single detachable coil and outcomes. AJNR Am J Neuroradiol 2005;26:1916-20. 